govt-data-11626227610032.html. Updated July 21, 2021. Accessed August 26, 2021.

6. COVID-19 pandemic: treatment protocol for patients with coronavirus infection. Tamil Nadu government website. https://cms.tn.gov.in/sites/ default/files/go/hfw_e_233_2021.pdf. Published May 10, 2021. Accessed August 26, 2021.

7. Soumyadeep B, Oommen J, Vivekanand J. Low-value medical care in the pandemic - is this what the doctor ordered? Lancet Global Health 2021; 9:E1203-E1204.

8. Staats CC, Kmetzsch L, Schrank A, Vainstein MH. Fungal zinc metabolism and its connections to virulence. Front Cell Infect Microbiol 2013;3:65.

9. Leonardelli F, Macedo D, Dudiuk C, Theill L, Cabeza MS, Gamarra S, Garcia-Effron G. In vitro activity of combinations of zinc chelators with amphotericin B and posaconazole against six mucorales species. Antimicrob Agents Chemother 2019;63:e00266-19.
10. RECOVERY Collaborative Group. Azithromycin in patients admitted to hospital with COVID-19: a randomised, controlled, open-label, platform trial. Lancet 2021;397:605-612.

11. Mucormycosis epidemic in India: out of the box menu. CME Media website. https://cmeindia.in/mucormycosis-epidemic-in-india-out-of-the-box-menu/ . Published May 24, 2021. Accessed August 26, 2021.

12. Mehta A, PandeyA. Rhino-orbital mucormycosis associated with COVID-19. Cureus 2020;2:e10726.

13. Belmonte JA, Dominguez-Sampedro P, Perez E, Suelves JM, Collado JM. Severe burns related to steam inhalation therapy. Anales de Pedatria 2015;82:95-99.

14. Nagarajan R. COVID-19: shortage of black fungus drug amphotericin-B looming. Times of India website. https://timesofindia.indiatimes.com/ india/covid-19-shortage-of-black-fungus-drug-looming/articleshow/ 83079300.cms. Published May 30, 2021. Accessed August 26, 2021.

\title{
Coronavirus disease 2019 (COVID-19) is a healthcare dilemma for human immunodeficiency virus (HIV)-positive individuals in Pakistan
}

\author{
Ali Raza DVM¹, Irfan Ullah MBBS², Muhammad Junaid Tahir MBBS ${ }^{3}$, Abdul Jabbar MPhil ${ }^{4}$ (1) and Ali Ahmed MPhil ${ }^{5}$ \\ ${ }^{1}$ Faculty of Veterinary Sciences, University of Veterinary and Animal Sciences, Lahore, Punjab, Pakistan, ${ }^{2}$ Kabir Medical College, Gandhara University, Peshawar, \\ Pakistan, ${ }^{3}$ Lahore General Hospital, Lahore, Punjab, Pakistan, ${ }^{4}$ Department of Clinical Medicine, Faculty of Veterinary Science, University of Veterinary and \\ Animal Sciences, Lahore, Punjab, Pakistan and ${ }^{5}$ School of Pharmacy, Monash University, Jalan Lagoon Selatan, Bandar Sunway, Subang Jaya, Selangor, \\ Malaysia
}

To the Editor-Coronavirus disease 2019 (COVID-19) has been spreading worldwide since the first case was reported in Wuhan, China, in early December 2019. The outbreak was declared a pandemic by the World Health Organization (WHO) on March 12, 2020. On February 26, 2020, the Ministry of Health, Government of Pakistan, confirmed the first case of COVID-19 in Karachi. ${ }^{1}$

According to the Centers for Disease Control and Prevention (CDC), a syndemic is defined as "synergistically interacting epidemics," that is, 2 or more concurrent or sequential epidemics or disease clusters in a community, which exacerbates the prognosis and burden of disease. Although the Asia and the Pacific region has 5.8 million human immunodeficiency virus (HIV)-positive patients and the coronavirus disease 2019 (COVID-19) pandemic has spread across Asia and the Pacific region, limited data are available on infection among HIV-positive patients. The acquired immunodeficiency syndrome (AIDS) caused by HIV has become a severe public health issue worldwide, particularly in underdeveloped nations. Approximately 37.8 million people are living with $\mathrm{HIV}$, and in 2020, 680,000 people died from AIDS-related illnesses globally. In Pakistan, 200,000 individuals are infected with HIV. ${ }^{2}$ The Joint United Nations Program on HIV/AIDS (UNAIDS) is in charge of the global campaign to eradicate AIDS as a public health hazard by 2030 . A new report from the

Author for correspondence: Abdul Jabbar, E-mail: vet.drabduljabbar@gmail.com Cite this article: Raza A, et al. (2022). Coronavirus disease 2019 (COVID-19) is a healthcare dilemma for human immunodeficiency virus (HIV)-positive individuals in Pakistan. Infection Control \& Hospital Epidemiology, 43: 1735-1736, https://doi.org/ 10.1017/ice.2021.376
Global HIV Prevention Coalition, which summarizes the state of HIV prevention programs during the COVID-19 pandemic, highlights significant vulnerabilities, dangers, and severe service interruptions. However, HIV service improvements and adjustments are achievable. ${ }^{3}$

The UNAIDS Global AIDS program provides evidence that HIV-positive persons are more vulnerable to COVID-19, which can be prevented by getting COVID-19 vaccination and HIV treatment. The new report details how lockdowns due to COVID-19 and other restrictions have severely hampered HIV testing, resulting in sharp reductions in HIV diagnoses, care providers, and treatment initiations in several countries. ${ }^{4}$ The UNAIDS Deputy Executive Director for Programs Shannon Hader stated that COVID-19 has disproportionately affected HIV prevention programs and cultural obstacles for the individuals most left behind, such as critical populations, teenaged girls, and young women. ${ }^{3}$

Despite a global decline in the number of new HIV infections, Pakistan is seeing an increase in the number of HIV cases. ${ }^{5}$ Pakistan has had several HIV outbreaks since 2003, making it Asia's second fastest-rising HIV nation. ${ }^{6}$ A variety of socioeconomic factors, such as poverty, illiteracy, and unemployment, likely contribute to HIV transmission in Pakistan. ${ }^{7}$ Also, HIV infection involves male sex workers (MSWs) rather than female sex workers, which indicates the variation in regional sex practices in Pakistan. AIDS infections among intravenous drug users (IDUs) are also increasing; IDUs are a potential source of HIV transmission because users share drugs and needles and can pass the virus on to sexual partners, resulting in HIV replication. Pakistan appears to be following the "Asian Epidemic" model as a result., 
In Pakistan, the National AIDS Control Program has registered $240,000 \mathrm{HIV}$ cases, and $55.62 \%$ of these patients on antiretroviral therapy (ART) in 50 ART centers. ${ }^{9}$ The main reasons reported for the late initiation of HIV treatment were feeling well and not experiencing symptoms, lack of willingness to communicate their HIV test findings, and fear of stigma and prejudice in their community. ${ }^{7}$ According to program statistics from Nai Zindagi, a nongovernmental organization (NGO) that provides harm-reduction services to IDUs in 38 districts of Pakistan, the HIV prevalence of HIV among IDUs reached $46.1 \%$ between 2011 and $2019 .{ }^{10}$ To eliminate the AIDS pandemic by 2030, UNAIDS has adopted a fast-track approach that aims to achieve the 95-95-95 goals by 2030: $95 \%$ of people living with HIV to know their status, $95 \%$ to be linked to ART treatment, and $95 \%$ of those in care to be virally suppressed. ${ }^{11}$ A high health-related quality of life (HRQoL) is a multifaceted term that incorporates independent, physical, spiritual, social, psychological, and environmental health of people living with HIV (PLWH). In Pakistan, only $14 \%$ of PLWH are aware of their HIV status; $10 \%$ of those infected are receiving treatment; and data on viral suppression are lacking. Pakistan is far from meeting UNAIDS 95-95-95 target. PLWH who are receiving ART have good HRQoL, but they report being significantly depressed. ${ }^{12}$

COVID-19 has caused widespread health-system disruption, leading to the cancellation of many regular health treatments in Pakistan. All resources and infrastructure dedicated to HIV/AIDS management have been transferred to the COVID-19 pandemic. PLWH are more likely to suffer from comorbid diseases and to be older; thus, they are more vulnerable to COVID-19. ${ }^{13}$ Data regarding how SARS-CoV-2 and HIV coinfection influence the health of PLWH are also scarce. ${ }^{14}$ PLWH in the United States, the United Kingdom, and South Africa have an $80 \%$ greater risk of death from COVID-19 infection, ${ }^{15}$ but no data on PLWH with COVID-19 or fatalities caused by COVID-19 are available in Pakistan. ${ }^{10}$ However, COVID-19 also offers a significant challenge to PLWH in Pakistan, where the threat of HIV is increasing and ART uptake is not promising. ${ }^{14}$ Quarantine, social distance, and community confinement have limited access to routine HIV testing and have hindered HIV care and ART continuation. In the context of the COVID-19 pandemic, PLWH face additional biological and social vulnerabilities, especially in settings where they suffer stigma and prejudice as well as inadequate healthcare services. ${ }^{14}$ Better care for this population can be achieved through proper health policies, pharmacist intervention, awareness, timely research, and evidencebased action. Interpersonal communication and mass media can be used to raise awareness about the prevention and control of HIV/AIDS, and interpersonal communication is more successful than mass media at doing so. ${ }^{16,17}$

The COVID-19 vaccine should be more widely available to people with underlying diseases, but unfortunately Pakistan, a lower- to middle-income country (LMIC), is having difficulty vaccinating people. ${ }^{18}$ Public awareness efforts about personal preventive measures should be encouraged among PLWH to prevent syndemic effects. To control all of the elements of the outbreak and to manage the country's increasingly serious health crisis, physicians, public health professionals, and the policy makers must work together.

\section{Acknowledgments.}

Financial support. No financial support was provided relevant to this article.
Conflicts of interest. All authors report no conflicts of interest relevant to this article.

\section{References}

1. Ali A, Ahmed M, Hassan N. Socioeconomic impact of COVID-19 pandemic: evidence from rural mountain community in Pakistan. J Public Affairs 2020. doi: 10.1002/pa.2355.

2. Bashandy SA, El Awdan SA, Ebaid H, Alhazza IM. Antioxidant potential of Spirulina platensis mitigates oxidative stress and reprotoxicity induced by sodium arsenite in male rats. Oxidat Med Cell Longev 2016. doi: 10.1155/ 2016/7174351.

3. Ahangarpour A, Oroojan AA, Alboghobeish S, Khorsandi L, Moradi M. Toxic effects of chronic exposure to high-fat diet and arsenic on the reproductive system of the male mouse. J Fam Reprod Health 2019; 13:181.

4. Khairul I, Wang QQ, Jiang YH, Wang C, Naranmandura H. Metabolism, toxicity, and anticancer activities of arsenic compounds. Oncotarget 2017; $8: 23905$.

5. Ahmed A, Saqlain M, Akhtar N, et al. Translation and cross-cultural adaptation of WHOQOL-HIV Bref among people living with HIV/AIDS in Pakistan. Health Qual Life Outcomes 2021;19:1-11.

6. Sharma O, Sultan AA, Ding H, Triggle CR. A review of the progress and challenges of developing a vaccine for COVID-19. Front Immunol 2020; 11:2413.

7. Ali H, Zakar R, Junaid K, Khan A, Fischer F. Frequency and reasons for delayed treatment initiation after HIV diagnosis: cross-sectional study in Lahore, Pakistan. BMC Public Health 2021. Doi: 10.1186/s12889-02111031-0.

8. Ahmed A, Hashmi FK, Khan GM. HIV outbreaks in Pakistan. Lancet HIV 2019;6:e418.

9. Milton AH, Hussain S, Akter S, Rahman M, Mouly TA, Mitchell K. A review of the effects of chronic arsenic exposure on adverse pregnancy outcomes. Int J Environ Res Public Health 2017;14:556.

10. Shah SA, Altaf A, Vermund SH. Challenges of antiretroviral treatment for human immunodeficiency virus infection in Pakistan. J Pakistan Med Assoc 2021;71:788-790.

11. Zubair M, Ahmad M, Saleemi MK, et al. Sodium arsenite toxicity on hematology indices and reproductive parameters in Teddy goat bucks and their amelioration with vitamin C. Environ Sci Pollut Res 2020;27: 15223-15232.

12. Ahmed A, Saqlain M, Bashir N, et al. Health-related quality of life and its predictors among adults living with HIV/AIDS and receiving antiretroviral therapy in Pakistan. Qual Life Res 2021;30:1653-1664.

13. Zhu F, Cao Y, Xu S, Zhou M. Reply to Comments on 'Coinfection of SARS-CoV-2 and HIV in a patient in Wuhan city, China.' J Med Virol 2020; 92:1417-1418.

14. Adadi P, Kanwugu ON. Living with HIV in the time of COVID-19: a glimpse of hope. J Med Virol 2020;10.

15. Ssentongo P, Heilbrunn ES, Ssentongo AE, et al. Epidemiology and outcomes of COVID-19 in HIV-infected individuals: a systematic review and meta-analysis. Sci Rept 2021;11:6283.

16. Yasin Z. Role of interpersonal communication and mass media in awareness, prevention and control of HIV/AIDS in Punjab, Pakistan. J Media Stud 2021;36:1.

17. Ahmed A, Rehman IU, Hong ACL, Hashmi FK, Awaisu A, Chaiyakunapruk N. Effect of pharmacist care on clinical outcomes among people living with HIV/AIDS: a systematic review and meta-analysis. Res Soc Admin Pharm 2021. doi: 10.1016/j.sapharm.2021.07.020.

18. Upadhyay P, Mehmood Q, Jabbar A, Ullah I, Siddiqi AR, Tahir MJ. Disproportionate COVID-19 vaccine distribution-a big threat for low-and middle-income countries. Infect Control Hosp Epidemiol 2021. doi: $10.1017 /$ ice.2021.320. 